\title{
ALFABETIZACIÓN ACADÉMICA: ESCRITURA Y LECTURA EN LA ENSEÑANZA DE ANTROPOLOGÍA DE LA EDUCACIÓN
}

ACADEMIC LITERACY: READING AND
WRITING IN THE ANTHROPOLOGY
OF EDUCATION TEACHING

Juan Carlos Mijangos Noh Ilse Maribel Ambrocio Santamaría

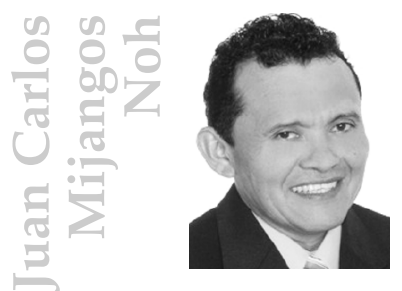

Licenciatura en Ciencias Antropológicas, Facultad de Ciencias Antropológicas, Universidad Autónoma de Yucatán. Maestría en Antropología Social, Facultad de Ciencias Antropológicas, Universidad Autónoma de Yucatán. Doctorado en Ciencias de la Educación, Universidad de La Habana, Cuba. Posdoctorado en Alfabetizaciones Académicas y Escritura a través del Currículum, Facultad de Filosofía y Letras, Universidad de Buenos Aires. SNI. Correo electrónico: [jc.mijangos@uady.mx].
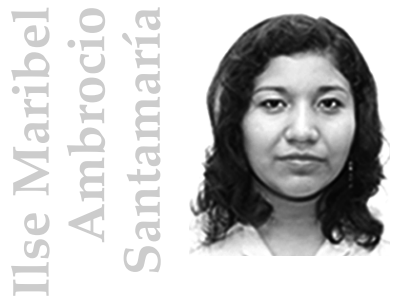

Estudiante del programa de Licenciatura en Educación que se imparte en la Universidad Autónoma de Yucatán.

Correo electrónico: [ilseambri@gmail.com].

\section{RESUMEN}

Este trabajo analiza, desde un enfoque de la teoría de alfabetización académica, diferentes estrategias empleadas en un curso de Antropología de la Educación, destinadas a mejorar la lectura crítica y la escritura de los participantes. El estudio se realizó en una universidad pública mexicana, específicamente con los estudiantes de tercer semestre en un programa de Licenciatura en Educación. Los datos 
recogidos y analizados mediante métodos etnográficos, crean una perspectiva complementaria y presumiblemente integral del maestro y de los puntos de vista de cada uno de los estudiantes, quienes son co-autores de este documento. En las conclusiones se publican los resultados obtenidos por medio de las estrategias empleadas a lo largo del curso y se establecen temas de investigación para estudios posteriores.

Palabras clave: alfabetizaciones académicas; habilidades de escritura y lectura.

\begin{abstract}
In this article we examined, based on the theoretical perspective of academic literacy, various strategies used in a course of Antropología de la Educación (AE) with the aim of improving the abilities of critical reading and academic writing of the participant students. The study had place in a public Mexican university, specifically in students of the third semester in an education degree. The information was collected and analyzed with ethnographic methods, generating a complementary perspective and holistic aspiration starting from the teacher's points of view and the observations of a student, both co-authors of this document, plus the interviews made to the participants of the course. In the conclusions are located the results obtained with help of the strategies used along the course and are established potential investigation subjects for subsequent research.
\end{abstract}

Key words: academic literacies; writing and reading skills.

\title{
EL CONCEPTO DE ALFABETIZACIÓN ACADÉMICA
}

\author{
Según Costanza Padilla y Paula Carlino:
}

El concepto teórico de «alfabetización académica», acuñado en Inglaterra y utilizado en plural para destacar la diversidad de prácticas escriturales y las relaciones de poder implicadas en ellas (Lea y Street, 1998), se ha 
extendido para abarcar en forma más general la formación para escribir en la universidad y las disciplinas ${ }^{1}$.

Para Carlino ${ }^{2}$, las alfabetizaciones académicas constituyen el conjunto de procesos merced al cual se establecen estrategias y nociones que permiten a un neófito hacerse partícipe de la cultura discursiva de alguna disciplina. En ese sentido, apunta la misma autora, estos procesos implican procesos de apropiación de las formas de lenguaje y pensamiento considerados propios y apropiados en el ámbito académico. El producto final de dichos procesos de alfabetización sería, entonces, el adquirir la pertenencia a una comunidad profesional, científica o académica. Según Carlino, estos procesos también son reconocidos como procesos de alfabetización superior o terciaria.

En un estudio dedicado al análisis de los procesos de alfabetizaciones académicas en universidades australianas y de los Estados Unidos, Carlino apunta que en dichas instituciones la lectura y la escritura son conceptualmente apreciadas en las formas que a continuación se describen:

a) Tanto la lectura como la escritura son necesarias para aprender a pensar críticamente dentro de los marcos y conceptos disciplinares.

b) El dominio de las prácticas discursivas de una disciplina es condición indispensable para el ingreso a una cultura académica.

c) Es necesario formar las capacidades para interpretar y producir textos especializados. Dichas capacidades deben formarse y no se alcanzan de manera espontánea ${ }^{3}$.

Desde el punto de vista de la misma autora, el desarrollo de las anteriores habilidades de escritura requiere del novicio diversos

1 C. Padilla, y P. Carlino, «Alfabetización académica e investigación acción: enseñar a elaborar ponencias en la clase universitaria», Alfabetización académica y profesional en el siglo XXI: leer y escribir desde las disciplinas, p. 2.

2 P. Carlino, «Alfabetización académica: un cambio necesario, algunas alternativas posibles», Educere. La Revista Venezolana de Educación, p. 410.

3 Ibidem, p. 416. 
aprendizajes, entre los que destacan: al escribir, la capacidad de tener presente al lector potencial; ser capaz de revisar textos escritos en forma sustantiva, desarrollando así la capacidad de transformar el saber inicial, lo cual solamente se consigue en un proceso complejo de armar y desarmar ideas, textos y argumentos en una fragua lenta y laboriosa ${ }^{4}$.

En una actualización de las ideas antes expuestas, Carlino plantea que:

La alfabetización académica es el intento por todos los medios de incluir a los alumnos en la cultura universitaria de cada asignatura. Es el conjunto de acciones que debemos realizar los docentes, con apoyo y orientación de la universidad, para que nuestros alumnos puedan leer, escribir, estudiar, comprender, pensar, argumentar, discutir, exponer, valorar razonamientos, debatir, etc., según los modos propios de hacerlo en cada disciplina ${ }^{5}$.

Por su parte, Caldera y Bermúdez ${ }^{6}$ asumen la alfabetización académica como las estrategias y actividades de escritura y lectura con que, en cada disciplina, se construye el conocimiento y se constituyen prácticas discursivas propias. En ese sentido, de acuerdo con estos autores, el aprendizaje y producción de la escritura implica la aplicación de estrategias en cada momento que involucra la creación del texto. Estos procesos se orientan por normas, costumbres y prácticas propias de cada campo disciplinario ${ }^{7}$.

Es a la luz de estas ideas teóricas, y del método que a continuación se describe, que recabamos y examinamos los datos recogidos en este estudio.

4 P. Carlino, «El proceso de escritura académica: cuatro dificultades de la enseñanza universitaria», Educere. La Revista Venezolana de Educación, p. 324.

5 P. Carlino, «Ingresar y permanecer en la universidad pública». El Eco de Tantil, p. 5.

6 R. Caldera y A. Bermúdez, «Alfabetización académica: comprensión y producción de textos», Educere. La Revista Venezolana de Educación, p. 248.

7 Ibidem, p. 253-254. 


\section{Método}

La investigación que se reporta se realizó de agosto de 2011 a marzo de 2012, en una facultad de educación de una universidad pública mexicana. Concretamente, el estudio se originó en el marco de la asignatura Antropología de la Educación (AE), impartida a estudiantes del tercer semestre del programa. El grupo de participantes fue de 26 personas: 20 mujeres y 6 hombres, cuyas edades oscilaban entre los 19 y 25 años.

Para la recolección de datos se emplearon técnicas y métodos asociados con el proceso etnográfico; específicamente, la observación participante ${ }^{8}$, la entrevista etnográfica ${ }^{9} \mathrm{y}$ el análisis etnográfico de contenido ${ }^{10}$.

La observación participante se realizó a partir de dos puntos de vista diferentes y complementarios: el del docente y el de una estudiante que fungía como co-investigadora ${ }^{11}$ (y, en consecuencia, aparece como co-autora de este artículo). Esto proporciona una visión más holística, o por lo menos bidimensional, del fenómeno estudiado.

Las entrevistas ofrecieron rica y abundante información respecto de la forma en que los estudiantes percibían, concebían, se explicaban a sí mismos y actuaban respecto de las dificultades, problemas y aprendizajes que experimentaban a lo largo del proceso de escritura. En ese sentido, constituyen un método que se complementa con el análisis etnográfico de contenido a través del cual, como se ve inmediatamente después de estas líneas, se obtuvo información profusa para este estudio.

Mediante el método de análisis etnográfico de contenido se analizó un total de 162 versiones de los trabajos finales, en distintas etapas del

8 J.P. Goetz y M. Lecompte, Etnografía y diseño cualitativo en investigación educativa, p. 125-127.

9 M.K. Gee y C. Ullman, «Teacher/Ethnographer in the Workplace: Approaches to Staff Development», Education Research Information Center, p. 1-2.

10 D. Altheide, «Ethnographic Content Analysis», Qualitative Sociology, p. 67.

${ }_{11}$ N. Santiago, «Research terminology: Subjects, participants, or co-researchers?», Education and Schools, 22-VIII-2010. 
proceso de creación. Lo anterior no quiere decir que se revisaran 162 trabajos diferentes, sino que se examinó el trabajo de cada uno de los 26 estudiantes en diversas ocasiones y fases del proceso de escritura; lo que se traduce en que el trabajo de cada uno fue examinado, en promedio, seis veces. Sobre la base del método antes descrito se obtuvieron los resultados que a continuación se describen.

\section{Resultados}

El trabajo principal con el cual se evaluó el curso, consistió en realizar una investigación antropológica y, consecuentemente, escribir un artículo cuyo proceso de desarrollo inició prácticamente desde el arranque del curso. El planteamiento subyacente a la escritura del artículo era que éste debía tomar en cuenta a la comunidad científica como lectora potencial de los textos producidos por los estudiantes. Lo anterior se apegó a las ideas teóricas planteadas en la primera sección de este trabajo.

De tal suerte, los requisitos de evaluación se acoplaron a los cánones de forma y contenido empleados en el ámbito concreto de la literatura educativa, más concretamente, en el ámbito de la AE. En otras palabras, la alfabetización académica a la cual se atendió en este caso, se enmarca en el ámbito de las ciencias educativas.

Para el desarrollo del escrito con el cual se evaluó el curso, se solicitó a los estudiantes emplear los cánones formales consignados en el Manual de estilo de publicaciones de la American Psychological Association (APA). A este respecto, una de las dificultades que tuvieron los estudiantes en cuanto al uso del Manual de la APA fue que observaron discrepancias respecto de lo que diferentes profesores enseñaron, antes de la asignatura $\mathrm{AE}$, sobre dicho documento normativo de las formas estilísticas en el campo educativo. Algunos profesores, en clases previas, incluso llegaban a plantear instrucciones o nociones que contravenían las indicaciones del Manual de la APA, con lo cual los estudiantes tenían motivos y causas de confusión.

Otra de las dificultades que un grupo importante de estudiantes presentó, de hecho la mayoría, fue respecto del desarrollo de la 
capacidad para la construcción de argumentos e ideas. El problema antes descrito se relaciona con las habilidades de lectura crítica. Al principio del curso de $\mathrm{AE}$, los estudiantes mostraron síntomas de no poder construir argumentos sólidos puesto que, durante las primeras lecturas realizadas en clase, al solicitar el docente a los estudiantes que opinaran acerca del tema de lectura, en varias ocasiones los participantes guardaron silencio sin saber qué decir. Posteriormente, la situación fue cambiando y en las clases finales se observó que participaban y opinaban, argumentando sus ideas adecuadamente. En esta transformación influyeron algunas de las estrategias de lectura que más adelante se describen y que atendieron al problema de comprensión de lectura examinado en el párrafo siguiente.

Desde el primer día del curso se realizaron lecturas que requerían comprensión de los temas relativos a la Antropología en general y a la AE en particular. Durante los primeros días del curso, la mayoría de los estudiantes demostraron no entender las lecturas pero posteriormente, y con la práctica repetida de las mismas, se logró la comprensión respecto de aquellos textos que describían características y aspectos teóricos relevantes de la AE. En otras palabras: fueron capaces de apropiarse de los fundamentos teóricos de la AE, sin los cuales el desarrollo del trabajo final con el que se evaluó el curso hubiera resultado imposible. De nueva cuenta, este proceso no ocurrió de manera espontánea: fue resultado de la instrumentación de procedimientos de lectura y apoyo al pensamiento crítico referidos y explicados más adelante.

Además de los dos problemas antes descritos, los estudiantes también padecían por el desconocimiento sobre el empleo de bases de datos y fuentes científicas legítimas y legitimadas en el ámbito de las ciencias educativas. De hecho, puede afirmarse que el empleo de bases de datos y fuentes científicas fue una de las dificultades más significativas para la elaboración del trabajo final, ya que al principio del curso la mayoría de los estudiantes no sabía dónde encontrar textos confiables que fundamentaran sus escritos. Desde el punto de vista de las alfabetizaciones académicas, esto constituye un problema medular, pues las formas canónicas de escribir en un área disciplinar específica se aprenden sobre la base de la lectura y el estudio de materiales que la comunidad científica pone en común, por medio de espacios 
socioculturalmente legitimados. En este caso, como en otros, las bases de datos que ofrecen materiales que la comunidad científica reconoce como apropiados — producto de procesos rigurosos de verificación, crítica y certificación-, son muy específicas y poseen características que nadie que pretenda ingresar a una comunidad académica, universitaria o científica ${ }^{12}$ debe o siquiera puede ignorar.

Otro problema del que adolecían los estudiantes del grupo investigado se relacionaba con la ortografía y gramática. Al principio de la redacción del texto científico, los estudiantes mostraron síntomas claros que evidenciaban problemas de ortografía y gramática: acentuación, dificultades para el uso correcto de los indicadores de género y número, para el empleo apropiado de tiempos y modos verbales, dificultades para reconocer las formas apropiadas de uso de las letras mayúsculas y, también, problemas con el empleo de los diferentes signos de puntuación. Se puede afirmar que los estudiantes desconocían $\mathrm{y}$, por lo tanto no aplicaban, las reglas ortográficas y gramaticales; fue necesario recordarlas pero, sobre todo, generar una disciplina para el empleo de los instrumentos que para tales fines provee la instancia que regula el uso culto del español: la Real Academia Española (RAE). A este respecto se describen, líneas abajo, las estrategias suscitadas en el proceso de enseñanza.

Por otra parte, los estudiantes mostraron un notable desconocimiento de conceptos relacionados con el campo disciplinario (en general el de las ciencias educativas y, en particular, el de la AE). Si bien es cierto que esto era de esperar - dado que precisamente el curso de AE tiene por objetivo proveerlos de las herramientas conceptuales y teóricas propias de su ámbito-, este problema se vincula con el anteriormente descrito, relativo a las dificultades al realizar una correcta lectura crítica de textos. Durante las clases, en varias ocasiones fue notorio en los estudiantes el desconocimiento respecto a conceptos relacionados con el campo disciplinario de estudio. Por ejemplo, cuando se hacía una pregunta a los participantes, referente a

12 P. Carlino, «Alfabetización académica: un cambio necesario, algunas alternativas posibles», Educere. La Revista Venezolana de Educación, p. 4-5. 
la asignatura o los textos, eran incapaces de responderla porque no entendían algunos conceptos propios de aquella asignatura o texto. Esto se relaciona con la falta de hábitos de búsqueda de conceptos y términos especializados en diccionarios, enciclopedias y demás materiales de consulta del ámbito educacional; luego entonces, nos encontramos ante un fenómeno que sí constituye una dificultad mayor: la falta de comprensión de lectura. Como más adelante se examinará, este problema también constituyó el motivo de la aplicación de estrategias para solucionarlo.

El desconocimiento del idioma inglés constituyó otra dificultad para los estudiantes observados puesto que, en la universidad, se espera que los aspirantes a la nueva cultura universitaria lean al menos en un idioma distinto del propio (en específico, el idioma inglés). El problema se suscitó debido a que, dentro de la bibliografía proporcionada por el profesor para aprender conceptos básicos sobre $\mathrm{AE}$, se indicó una lectura en ese idioma y cuando se revisó dicho texto de manera colectiva, sólo unos cuantos estudiantes del grupo sabían leer en inglés. Esto añadía un elemento más al problema de comprensión lectora.

La universidad es formadora de profesionales de áreas específicas; cada una de éstas posee una cultura académica a la cual los recién ingresados desean y deben acceder para una formación exitosa. Para lograr la incorporación de los nuevos estudiantes universitarios a la cultura discursiva del caso estudiado, fue necesario considerar los aspectos de cultura académica que esa universidad y esa facultad, en particular, creen relevantes para la formación de discentes. A continuación, se describirán algunas peculiaridades de la cultura académica promovida en el caso de estudio que aquí se reporta.

En la universidad, la lectura y escritura se consideran elementos consustanciales a la labor educativa, ya que por medio suyo se pretende la formación de profesionales críticos, con una educación continua y autónoma que propicie el autoaprendizaje. Se puede afirmar que lo planteado anteriormente es posible con la adecuada lectura crítica y por medio del empleo de la escritura como forma de crear conocimientos estructurados y consolidados. En otras palabras, la 
formación de profesionales de la educación capacitados en la lectura y el uso disciplinariamente correcto del lenguaje escrito es un objetivo que pretende alcanzar la universidad.

Una de las principales peculiaridades de este caso es que la asignatura $\mathrm{AE}$ es obligatoria dentro del plan de estudios de la Licenciatura en Educación; tiene un valor de ocho créditos y se ofrece a partir del tercer semestre. En ese sentido, puede señalarse que es una materia considerada importante para la formación del licenciado en Educación, ya que requiere que los estudiantes aprendan conceptos relevantes, precisos y pertinentes para desarrollarse dentro de las ciencias educativas, comunicándose mediante conceptos apropiados y en un adecuado lenguaje, verbal y escrito.

La $\mathrm{AE}$ es relevante para el área de currículo, debido a la relación que guarda con el análisis y selección de contenidos culturalmente apropiados. Por lo tanto, quienes diseñaron el currículo de la Licenciatura en Educación, del caso que aquí se reporta, consideraron que dicha asignatura ayudaría al profesional en su formación, particularmente al brindarles elementos para la adecuada apreciación de la cultura de los discentes y las características de pertinencia, respecto a los contenidos curriculares y a los medios propuestos para su enseñanza.

Otro requisito de gran relevancia en la universidad donde se efectuó el estudio de caso para la integración del estudiantado a su cultura, es la adecuada expresión dentro de los cánones establecidos en las disciplinas que conforman el currículo de las ciencias educativas. En el caso estudiado se pretendió que los estudiantes utilizaran términos del campo de la $\mathrm{AE}$ y que principalmente, pero no solamente en el trabajo final, se expresaran adecuadamente, empleando conceptos propios de dicha disciplina y, al mismo tiempo, construyeran argumentos originales acordes con los cánones de dicho campo disciplinario en particular.

En la universidad también se espera que los estudiantes utilicen fuentes y documentos especializados, relacionados con la disciplina de estudio para la correcta formación de los profesionales. Sin 
embargo, al inicio del curso de AE, distintos estudiantes manifestaron que se les dificultaba la localización, lectura, valoración y el empleo crítico de documentos especializados. Para contrarrestar esta problemática, en el proceso de alfabetización académica que se desarrolló en el caso investigado, el profesor señaló como un criterio para evaluar el escrito final, el uso de fuentes y documentos especializados en ciencias educativas, Antropología y en AE. Específicamente, brindó ejemplos concretos de búsqueda de documentos, modeló el proceso de lectura para sus estudiantes en sesiones de lectura colectiva y mostró cómo incorporar en dicho proceso el empleo de diccionarios especializados y otras fuentes de consulta.

En relación con los documentos escritos, en la universidad en la que se produjo el caso que aquí se reporta, se considera que el empleo de un tono y lenguaje apropiados a los usos de las disciplinas educativas es otro elemento formativo que los estudiantes deben cubrir. En otras palabras, se espera que los estudiantes se comuniquen de manera escrita con el lenguaje adecuado y el tono propio de las ciencias educativas; por tal razón, en el caso particular del estudio de $\mathrm{AE}$, se consideró dicho elemento como uno de los criterios de evaluación.

En el caso estudiado se considera esencial el uso del lenguaje dentro de los parámetros establecidos por una comunidad académica. En el ámbito de los cánones formales y estilísticos, los elementos descritos en el Manual de estilo de publicaciones de la American Psychological Association suelen ser los más empleados en el campo de la educación. El empleo del antedicho manual se contempla desde los primeros pasos de la formación de los estudiantes, por lo que se les empieza a involucrar en su aprendizaje y uso a partir del primer semestre del programa. Debe enfatizarse que, como se señala en otra parte de este texto, este aprendizaje (de los cánones formales de escritura dentro de la disciplina educativa) no está exento de contradicciones, pues algunos profesores de otras asignaturas no siguen, o no conocen, las prescripciones del manual de estilo aquí comentado. 


\section{Estrategias empleadas por el docente}

Para la inserción de los alumnos del caso estudiado, a la cultura académica universitaria - cuyos rasgos se han descrito-, el profesor estableció estrategias para subsanar las deficiencias en las habilidades respecto a la comprensión lectora y la correcta escritura. Dichas estrategias se llevaron a cabo durante las primeras sesiones, instruyendo claramente sobre aquello que se esperaba en la asignatura de $\mathrm{AE}$; esto fue de gran ayuda para los estudiantes para la realización del trabajo final. A continuación se describen algunas estrategias implementadas en el caso investigado:

\section{El amigo y el guardián}

Estas estrategias se implementaron a lo largo del curso. El profesor indicó que el producto final del curso sería una investigación antropológica realizada individualmente pero mediante acciones colaborativas. Esto brindó a cada estudiante la oportunidad de escoger a un "amigo», es decir, un compañero con quien se tuviera la suficiente confianza para ayudarle a evaluar y corregir errores durante el proceso de redacción, contando así con alguien para la elaboración de su trabajo. Además del amigo seleccionado por cada estudiante, el docente asignó a cada discente a un "guardián», es decir, a un par que cumpliría más o menos las mismas funciones que el amigo pero con un nivel mayor de responsabilidad administrativa, puesto que los errores detectados por el profesor en el trabajo escrito final no serían descontados al autor responsable de dicho trabajo, sino a su guardián.

Cuando los estudiantes comenzaron a trabajar en sus escritos, la falta de costumbre respecto de la revisión del texto y del empleo de diccionarios, manuales y demás fuentes de consulta general o especializada, dieron como resultado que los trabajos presentaran cantidades considerables de errores y carencias de diferente índole. Posteriormente, cada participante del curso se fue dando cuenta de los errores de su amigo y cada guardián se involucró con el trabajo escrito de su compañero $\mathrm{y}$, al mismo tiempo, amigos y guardianes fueron tomando conciencia de los propios errores al escribir. 
En la práctica, ambas estrategias - la del amigo y la del guardián- generaron una dinámica de revisiones múltiples que introdujeron a los estudiantes en aspectos poco enfatizados del proceso de alfabetización académica en la formación inicial de los profesionales de la educación. Esto empezó con el proceso de descubrimiento de que la escritura no es un ejercicio solitario sino social y colaborativo $y$, por otra parte, la escritura académica no puede hacerse improvisadamente, sino a costa de un laborioso y prolongado proceso de revisiones que contribuyen al perfeccionamiento y control de la calidad del texto, texto que tiene a un lector específico con características particulares social y culturalmente construidas.

Estudiantes participantes en el estudio sugieren que estas dos estrategias, la del amigo y la del guardián, fueron de gran ayuda. En particular, indican que fue más fácil corregir los trabajos de otros estudiantes que los propios; esto les permitió generar estrategias para mejorar la lectura crítica, la argumentación escrita, el tono científico y el estilo, esto último se hizo específicamente a través de la correcta utilización del manual de la APA en cuanto a: citas, referencias, márgenes, resumen, formato de letra, espaciado, cornisa, tipografía, etcétera. De hecho, las estrategias tuvieron efectos multiplicadores en la práctica, pues los estudiantes solían pedir a más de dos compañeros que revisaran sus trabajos. Con el incremento del número de revisores, también se generó un número mayor de lectores y lecturas del manual de la APA y otros materiales, como la ortografía y los diccionarios de la RAE y, en menor medida, la gramática y las lecturas especializadas en AE. Esto último, que bien podría evaluarse como una dificultad por subsanar, se atendió mediante la estrategia de tutoría presencial descrita más adelante.

\section{Tutoría virtual}

Como estrategia, consistió en brindar asesoría al estudiante por parte del profesor, mediante una lista de correo electrónico y mensajes particulares. Cada estudiante enviaba el trabajo al docente por medio de correo electrónico, al menos una vez por semana, así el profesor debía reenviárselo con las correcciones y comentarios 
necesarios; empero, el profesor no cambiaba el contenido, sólo realizaba observaciones de forma, estilo de la APA y redacción.

\section{Tutoría presencial}

Se realizó en las clases finales de la asignatura. El profesor llamaba a cada estudiante para revisar su trabajo y, a diferencia de la tutoría por correo electrónico, ésta se orientó más al contenido argumental del texto, porque los errores de esa índole se hacían notar a los estudiantes y se les explicaban formas de enmendarlos. Aclarado el error, los estudiantes procedían a la reconstrucción del trabajo y a la revisión con el amigo y el guardián.

\section{Construcción colectiva de los criterios de evaluación para el trabajo final}

A mitad del semestre, el docente pidió a los estudiantes que, mediante sus conocimientos previos y el análisis de los artículos científicos - que para aquel momento, ya se habían consultado-, determinaran los elementos que en el trabajo final deberían evaluarse. Esto se hizo con el fin de que los mismos estudiantes tuvieran un espacio para reflexionar sobre la cultura discursiva en la que estaban siendo instruidos y pudieran cumplir, en forma consciente, con los requisitos para aprobar la materia. Esta sesión fue complicada; se expresaron ideas confusas respecto de los requerimientos de un trabajo de investigación, se formaron grupos que planteaban alguna característica y otros que contradecían tal planteamiento. Al final se llegó a un consenso y el profesor procedió a aclarar y corregir las características de un trabajo de investigación en el campo de la AE. Así, las confusiones de los discentes fueron elucidadas.

Además de las estrategias antes descritas, el profesor instituyó estrategias asociadas al proceso de aprendizaje de la lectura crítica, con el fin de ayudar a los estudiantes en sus respectivos trabajos finales. 


\section{El uso de diccionarios especializados y de la lengua}

Como se había planteado, una de las problemáticas de los estudiantes era el desconocimiento de términos relacionados con la asignatura de AE. Para ayudarlos a involucrarse con esta disciplina, en cada clase, al realizar la lectura colectiva y encontrar conceptos desconocidos se les solicitaba que, en libros y diccionarios especializados proporcionados por el profesor, se consultaran los significados de dichos términos. Al principio esta estrategia era tediosa para ellos, sin embargo, al avanzar las clases, en muchas ocasiones sin que el profesor solicitara la consulta, los estudiantes la realizaban por cuenta propia. Dicha estrategia fue relevante ya que en la construcción del texto científico, cada estudiante la implementaba durante su búsqueda de literatura, brindándole una comprensión más profunda de los textos empleados.

\section{La lectura dirigida por el profesor}

En la universidad, la lectura es un factor principal para el aprendizaje pero, en algunas clases, no se practica recurrentemente o se realiza de manera deficiente. Por lo anterior, desde el punto de vista de los participantes, la estrategia de lectura dirigida debería emplearse en todas las clases. En el caso investigado, el ejercicio se realizó en la mayoría de las clases con lecturas correspondientes al programa de AE. En ese sentido puede señalarse que el aprendizaje de la disciplina se realizó mediante lecturas diversas y especializadas y, según el estudiantado, este análisis marcó una diferencia sustancial respecto de las estrategias de enseñanza de otros profesores. A decir de los participantes, esto genera conciencia del estudiante en relación con la importancia que la lectura desempeña en la universidad.

\section{La enseñanza de formas de cuestionar al texto escrito}

Esta estrategia tuvo diversos impactos en los discentes. Uno de ellos fue que se atrevieron a cuestionar a los autores de las lecturas, a partir de sus propios análisis, criterios y conocimientos. En un principio, los integrantes del grupo no se atrevían a hacerlo, al no considerar que fuera ésta una estrategia de lectura para la formación de sus 
propios conocimientos, considerando el conocimiento como un ente estático. Los estudiantes sentían inseguridad para cuestionar el texto, pero el profesor ejemplificó la manera de hacerlo y alentó a los estudiantes: en cada clase nombraba a un grupo para que formulara este tipo de cuestionamientos y, entre todos, llegar a una conclusión.

\section{Debate y argumentación de las ideas planteadas por los autores}

Esta estrategia guarda una relación estrecha con la antes descrita, sin embargo, posee rasgos particulares.

Al principio del curso, los estudiantes se quedaban callados cuando el profesor les pedía su opinión respecto a un tema específico relacionado con las lecturas del programa, en particular al debatir o argumentar a favor o en contra de las ideas expresadas por algún autor. Sin embargo, el profesor proporcionó ejemplos sobre cómo debatir y argumentar respecto de las ideas planteadas en los textos estudiados, de esta manera contribuyó a reforzar esta habilidad. También propició el debate durante las clases, involucrando y transportando al discente a su propia realidad para contrastarla con las ideas y planteamientos expresados en los textos. Este ejercicio específico de contrastar el planteamiento teórico con la realidad experimentada, brindó a muchos estudiantes niveles nuevos de involucramiento en el análisis de conceptos. Es decir, el cuestionamiento fue mucho más allá del examen respecto de la lógica interna del argumento: llegó a la ejecución de argumentos y contra-argumentos, ejercicio que es rasgo sine qua non del proceso de creación del conocimiento científico.

\section{Los rasgos característicos de los textos de una disciplina}

Esta enseñanza se logró mediante el análisis y la identificación de estos elementos en textos de AE. Dichos textos se especializaban en AE y coincidían en tener elementos en común, facilitando la identificación de los mismos. Una unidad completa del curso se dedicó a la lectura crítica y al análisis de casos de investigaciones que reportaban estudios de AE. Al final de cada caso, el profesor realizaba preguntas intercaladas para identificar los elementos clave, como: qué tipo de 
citas realizaba el autor, la bibliografía utilizada, las categorías de los subtemas y el título; al término de todos los casos los estudiantes, con ayuda del profesor, señalaron e identificaron los elementos que debía contener el texto del trabajo final.

\section{La lectura en otros idiomas}

Esta estrategia no consistió en enseñar otros idiomas, sino que fue un intento por comenzar a involucrar a los estudiantes en la lectura de textos en otros idiomas (en este caso, el inglés) que cumplieran con esta característica. Debido a que se les solicitó formular preguntas de las lecturas, en el caso de la lectura en inglés, se organizaron para que quienes leyeran en ese idioma ayudaran a la comprensión del texto; al final, la mayoría de los estudiantes pudo comprender de qué trató la lección. Por último, a este respecto puede decirse que los estudiantes tomaron conciencia de la importancia de leer en otros idiomas, pues para pertenecer a la cultura discursiva de las ciencias educativas es necesario leer y comprender otras lenguas, además de estar a la vanguardia con los descubrimientos efectuados en el campo de estas ciencias.

Otro tipo de estrategias que involucró el profesor para alfabetizar académicamente a los estudiantes fueron las asociadas con la administración de la información. Con este tipo de estrategias se pretendía ayudarlos a organizar su información facilitando su uso y búsqueda, además de utilizar información propia de las ciencias educativas.

\section{Búsqueda en bases de datos y fuentes científicas}

Anteriormente se mencionó que una de las problemáticas del estudiante para pertenecer a la cultura académica - específicamente aquélla asociada con la $\mathrm{AE}$-, era el desconocimiento de bases de datos y fuentes científicas convalidadas y reconocidas por la comunidad disciplinaria. Para enmendar esta problemática, el docente enseñó la utilización de ciertas bases de datos como la de la Red de Revistas Científicas de América Latina y el Caribe, y la base del Education Research Information Center. Posteriormente, 
los estudiantes empezaron la búsqueda de artículos que sirvieran para la fundamentación de su trabajo.

\section{La enseñanza de formas de organizar y archivar información}

Esta estrategia se implementó debido a que el profesor notó que algunos estudiantes no organizaban adecuadamente su información $\mathrm{y}$, al momento de solicitarles algún trabajo, extraviaban el mismo en su dispositivo USB o en los archivos de su computadora. Por lo tanto, para facilitar el uso de las lecturas proporcionadas, el profesor solicitó a cada uno crear una carpeta de la asignatura de AE en donde depositaran todas las actividades y lecturas realizadas en las clases así, al momento de requerir información, les sería más práctica y eficaz la recuperación de datos.

\section{La enseñanza de formas de tomar notas}

Esta estrategia consistió en enseñar a los estudiantes a tomar notas de las lecturas realizadas. Por ejemplo: cuando en clase se hacían lecturas colectivas, al terminar cada clase se pedía a algún estudiante que concluyera leyendo su síntesis escrita sobre el tema. El profesor cuestionaba y corregía los argumentos débiles e inexactos, ofrecía contraargumentos y pedía opiniones que se sumaran al debate. Esto ayudó a promover la construcción de argumentos y animaba a los estudiantes a tomar notas sobre aspectos importantes, ya destacados en el programa de estudios de la asignatura. También se debían tomar notas de las lecturas realizadas en la casa y en la clase siguiente se preguntaba acerca del texto leído.

Otro tipo de estrategias implementadas por el profesor de AE fueron las asociadas con el proceso del aprendizaje de la argumentación escrita. Ya que el producto final de la asignatura fue un artículo, el profesor debía ayudar al estudiante precisamente a argumentar de manera escrita, pues no se debe (aunque a menudo ocurre) solicitar y calificar la escritura si no se enseña cómo debe practicarse en la universidad. Este tipo de estrategias son importantes para la integración del estudiante a la cultura discursiva de la disciplina. Al mismo tiempo, 
ayuda a la creación del propio conocimiento y la comunicación del mismo. Es una de las estrategias olvidadas en las clases universitarias, donde se trata con mayor atención y frecuencia la comunicación verbal, muchas veces a expensas de la comunicación escrita.

\section{La enseñanza de la correcta ortografía y gramática}

La ortografía y gramática son aspectos que se enseñan en todos los niveles de la educación escolarizada. En el curso que se analiza y reporta en esta investigación se contempló como elemento de los criterios de evaluación para el trabajo final. Al ser así, a los estudiantes les fue indispensable cuidar la ortografía y gramática y, a diferencia de lo que suele ocurrir en otras asignaturas, los estudiantes de este grupo emplearon mayor dedicación a la creación del texto. Para afrontar esta problemática, el profesor proporcionó diccionarios de ortografía y gramática; algunas clases se dedicaron a recordar las reglas ortográficas; y en los intercambios de los trabajos con «el guardián», éste debía revisar esos aspectos, hasta el punto de conocer él mismo las características de la correcta ortografía y gramática. La enseñanza de estos elementos - si bien puede parecer un elemento secundario e incluso tenerse por sabido- constituye una de las principales ayudas para la adecuada comunicación escrita.

\section{La enseñanza del uso de las citas textuales y paráfrasis}

Antes de comenzar a redactar el texto científico que sería evaluado como trabajo final, el profesor solicitó a los estudiantes que crearan un anteproyecto en donde cada participante debía especificar un tema, los subtemas y la relación del tema con la AE. Para ello, los estudiantes debían auxiliarse de citas textuales, practicando la forma de presentación de cada cita, que posteriormente debía incorporarse en el trabajo; aunado a esto se construyó una lista de paráfrasis que ulteriormente debían emplearse en el escrito.

Esta enseñanza - de un perfil que suele ser ignorado a menudo por los docentes- constituyó para los estudiantes no solamente una dificultad importante, sino el reconocimiento de uno de los elementos más cuidados en el ámbito científico y profesional: evitar el plagio. 


\section{La enseñanza del proceso del desarrollo de las ideas}

Durante las lecturas y las revisiones del trabajo, el profesor realizaba observaciones, sugerencias y señalaba ideas que no tenían argumentación, propiciando que los discentes mejoraran cada vez el texto. Esto era complementario con el intercambio de ideas realizadas con el guardián y el amigo; de hecho se descubrió que, entre pares, existía más confianza para sugerir la mejora del trabajo y se tomaban en cuenta estas sugerencias en las correcciones. Con ese tipo de ejercicios como antecedente, los trabajos mejoraban sustancialmente y el aporte del docente podía hacerse en un nivel de precisión y detalle mucho más fino.

\section{Evidencia empírica y argumentos derivados de la autoridad científica}

Dado que el trabajo final solicitado fue el reporte de una investigación científica, eso supuso que todo artículo presentado debía ofrecer la evidencia empírica recabada. Con ese propósito, mediante ejemplos y en las tutorías presenciales, el docente señaló las maneras para presentar el trabajo de campo, supervisó los instrumentos aplicados durante la investigación e intervino en cada detalle de la investigación. Para este proceso, el profesor se auxilió con el análisis de las lecturas de $\mathrm{AE}$ - porque en éstas se ejemplificaba la manera de presentar evidencia empírica一, también sugirió lecturas sobre los métodos de investigación antropológica y educativa, para hacer uso del que fuera más apropiado para cada investigación.

\section{El desarrollo de la capacidad de síntesis}

Para favorecer el desarrollo de esta capacidad, el docente solicitó, como uno de los requisitos del trabajo final, que éste tuviera una extensión no mayor de 2,500 palabras (incluyendo: portadilla, resumen y referencias bibliográficas). Esto se hizo con el fin de que el estudiante analizara claramente cada cita textual y cada paráfrasis, discerniera entre la bibliografía consultada y optara por incorporar las ideas y evidencias más apropiadas para su tema de estudio. Mediante ese procedimiento, los estudiantes experimentaron la dificultad de sintetizar un tema específico en aproximadamente 
diez páginas, lo cual los obligó a discernir entre varios textos y datos, y apropiarse de los más relevantes para su trabajo.

\section{CONCLUSIONES}

El resultado del empleo de las estrategias anteriormente descritas fue el paulatino mejoramiento de los textos que sirvieron para evaluar el curso de AE que en este escrito se examina. Con esto se confirman muchos de los asertos planteados en la literatura sobre alfabetización académica publicada en América Latina ${ }^{13}$ (Caldera y Bermúdez, 2007; Carlino, 2011; 2004; 2003; Padilla y Carlino 2010).

Por otra parte, la experiencia deja ver algunas áreas de oportunidad que, en el caso estudiado, deben atenderse si se pretende elevar y mantener el nivel de calidad de los escritos de los estudiantes de la Licenciatura en Educación cuyo caso fue objeto de esta investigación. Dichas áreas de oportunidad apuntan a:

1. La necesidad de que los docentes se capaciten y sean modelos adecuados en el uso de las formas canónicas de escritura empleadas en el campo de las ciencias educativas.

2. La necesidad de considerar que dicho proceso, a fortiori, requiere de un arduo trabajo de revisión paulatina, constante y minuciosa de los escritos de los estudiantes, lo cual se traduce en enfatizar la calidad del proceso y los resultados, no en la cantidad de hojas y trabajos entregados.

13 R. Caldera y A. Bermúdez, «Alfabetización académica: comprensión y producción de textos», Educere. La Revista Venezolana de Educación, n. 037, junio, 2007, p. 247-255.

P. Carlino, «Alfabetización académica: un cambio necesario, algunas alternativas posibles», Educere. La Revista Venezolana de Educación, n. 020, enero-febrero-marzo, 2003, p. 409-420.

P. Carlino, «El proceso de escritura académica: cuatro dificultades de la enseñanza universitaria», Educere. La Revista Venezolana de Educación, n. 026, 2004, p. 321-327.

P. Carlino, «Ingresar y permanecer en la universidad pública», El Eco de Tantil, 30-IV2011, p. 5.

C. Padilla y P. Carlino, «Alfabetización académica e investigación acción: enseñar a elaborar ponencias en la clase universitaria», Alfabetización académica y profesional en el siglo XXI: leer y escribir desde las disciplinas, Academia Chilena de la Lengua-Ariel, Santiago de Chile, 2010, p. 153-182. 
3. La necesidad de modelar procesos de lectura crítica, proporcionando a los estudiantes las herramientas requeridas para el correcto ejercicio de esa habilidad.

4. La necesidad -no examinada en este artículo, pero detectada a lo largo de la investigación- de generar formas administrativas y de calificación que no sacrifiquen conocimiento y habilidad en aras de cumplir con los tiempos programáticos y las estadísticas de aprobación y reprobación. Esto significa que, actualmente, en algunas asignaturas se está aprobando a estudiantes que no cubren satisfactoriamente las habilidades de lectura y escritura que se supone deben adquirir, esto mediante el expediente de relajar el estándar de evaluación. Una asignatura con procesos como los descritos en este artículo no acude a ese expediente, mantiene un alto estándar, pero también requiere de más tiempo para la obtención de resultados que, habiéndose cumplido cabalmente con el proceso de aprendizaje, se traduzcan en calificaciones aprobatorias pero, sobre todo, en trabajos de buena calidad.

Todas estas conclusiones permiten atisbar, de alguna manera, nuevas oportunidades de investigación que esperamos retomar en trabajos posteriores. 


\section{REFERENCIAS BIBLIOGRÁFICAS}

ALTHEIDE, D., «Ethnographic Content Analysis», Qualitative Sociology, vol. 10, n. 1, 1987, p. 65-77.

CALDERA, R. y BERMÚDEZ, A., «Alfabetización académica: comprensión y producción de textos», Educere. La Revista Venezolana de Educación, n. 037, junio, 2007, p. 247-255.

CARLINO, P., «Alfabetización académica: un cambio necesario, algunas alternativas posibles», Educere. La Revista Venezolana de Educación, n. 020, enero-febrero-marzo, 2003, pp. 409-420.

«El proceso de escritura académica: cuatro dificultades de la enseñanza universitaria», Educere. La Revista Venezolana de Educación, n. 026, 2004, p. 321-327.

«Ingresar y permanecer en la universidad pública», El Eco de Tantil, 30-IV-2011, p. 5.

GEE, M.K. y ULLMAN, C., «Teacher/Ethnographer in the Workplace: Approaches to Staff Development», Education Research Information Center, ED423721, p. 1-2.

GOETZ, J.P. y LECOMPTE, M., Etnografía y diseño cualitativo en investigación educativa, Morata, Madrid, 1998.

LEA, M.R. y STREET, B.V., «The Academic Literacies Model: Theory and Applications», Theory into Practice, vol. 45, n. 4, otoño 2006, en: [http:/ / courses.essex.ac.uk/ia/ia409/E_pdf_copies_of_ readings/Lea_Street_intro_acad_litaracies.pdf]. 
PADILLA, C. y CARLINO, P., «Alfabetización académica e investigación acción: enseñar a elaborar ponencias en la clase universitaria», Alfabetización académica y profesional en el siglo XXI: leer y escribir desde las disciplinas, Academia Chilena de la Lengua-Ariel, Santiago de Chile, 2010, p. 153-182.

SANTIAGO, N. «Research terminology: Subjects, participants, or co-researchers?», Education and Schools, 22-IX-2010, en: [http: / / www.examiner.com/ scholarly-research-in-sanjose / research-terminology-subjects-participants-or-coresearchers]. 